\title{
From pneumomyelography to cord tractography: historical perspectives on spinal imaging
}
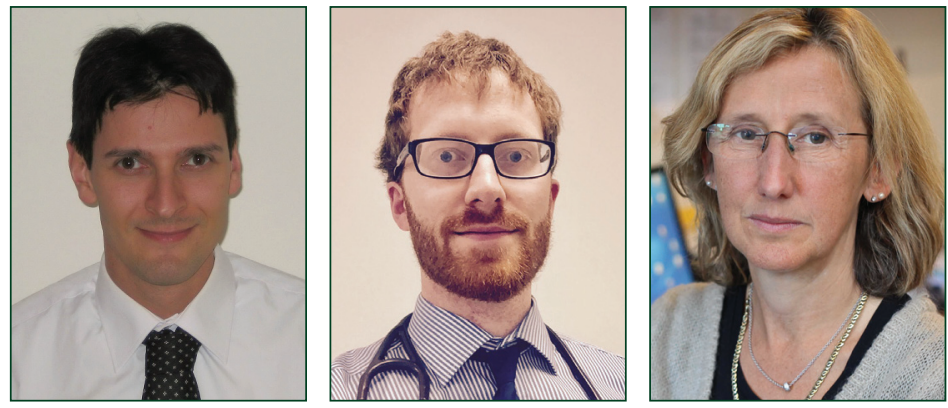

\section{“Despite recent developments, an urgent and unmet need persists for sensitive spinal measures.”}

Peter Bede ${ }^{*, 1}$, Eoin Finegan' \& Orla Hardiman¹

First draft submitted: 2 May 2017; Accepted for publication: 7 June 2017; Published online: 7 August 2017

Quantitative spinal cord imaging is one of the least explored frontiers of neuroradiology. While high resolution spinal cord imaging remains notoriously challenging, unprecedented technical advances have taken place in recent years foretelling exciting new clinical applications. The small cross-sectional area of the human cord, respiratory effects, cardiac pulsation and cerebrospinal fluid circulation are just some of the physiological factors making high quality spinal imaging significantly more difficult than brain imaging. Despite the anatomical and technical challenges, several MRI techniques previously only used in brain imaging, such as functional MRI and tractography, have recently been adapted to spinal applications.

Historically, spinal imaging has consistently lagged behind cerebral imaging. Vertebral $\mathrm{x}$-rays dominated the early years of spinal radiography and emerging singleplane methods in the 1930s, such as tomography or planigraphy, only offered limited additional value in characterizing osseous lesions. It was not until the development of contrast-enhanced techniques that the outline of the cord was successfully visualized. Pneumomyelography, myelography, discography, spinal angiograms and computed tomography myelography are just some of the pre-MRI methods which have been successfully utilized to visualize soft tissue structures in the spinal canal. The diagnostic value, ingenuity and elegance of these techniques are remarkable given the technological constraints of the pre-MRI era. All of these techniques were invariably invasive and relied heavily on the dexterity of neuroradiologists. Despite their limitations, the incessant optimization of these methods epitomizes the timeless drive to characterize pathology in vivo, either as part of a pre-surgical assessment or in an effort to elucidate unexplained neurological symptoms. At a time when specific spinal tracts can be evaluated with tractography, metabolic information gained from magnetic resonance spectroscopy (MRS), and spinal functional MRI captures segmental activation, a historical perspective serves as a reminder of the relentless technological advances.

\section{KEYWORDS}

- biomarker • MRI

- neurodegeneration

- neuroimaging $\bullet$ spinal cord 


\section{The advent \& development of} myelography

In Walter Dandy's original description of pneumoencephalography in 1919 there is a brief mention of visualizing the spinal cord contour following air injection [1]. In 1921 Hans Christian Jacobaeus and Sofus Widero proposed the use of pneumomyelography to diagnose spinal cord tumors [2]. The first contrast myleography is reported by Sicard and Forestier in 1922 [3]. Their use of iodized poppy seed oil (Lipiodol ${ }^{\circledR}$ ) was originally intended for intramuscular and epidural injections for sciatica until the accidental injection of Lipiodol intrathecally serendipitously outlined the spinal cord under fluoroscopy [4]. Following the success of lumbar myelography, Sicard and Forestier began injecting Lipiodol by occipital cisterna magna puncture. In addition to cisternal puncture, high cervical punctures at C1-C2 were also routinely performed for both gas and contrast myelography [5]. Gas myelography and iodinated oil contrasts were gradually replaced by Iophendylate in the 1940s and subsequently by water soluble noniodinated contrast agents in the 1960s. From the late 1970s CT myelography became the standard procedure to image acute spinal pathologies. Despite the widespread availability of MRI, CT myelography is far from obsolete. In patients with pacemakers, spinal rods, screws and wires, CT myelography remains an invaluable diagnostic tool. While the first cerebral angiography was reported in 1927 [6], the first spinal angiography was not performed until 1966 and it is not until the advent of subtraction techniques in the late 1960s that vascular malformations were reliably visualized. The first description of discography was published by Swedish radiologist, Lindblom in 1948. Discographies were initially performed at a lumbar level from a posterior, midline transdural approach, slowly perfected into a posterolateral technique applicable to thoracic and cervical regions [7].

\section{Current practice: methods, challenges \& limitations}

In current clinical practice, spinal MRI is the gold standard imaging modality in trauma, acute myelopathies, demyelination, suspected radiculopathy, lower back pain, and for detecting vascular malformations, tumors, syringomyelia and congenital malformations. While spinal MRI sequences successfully capture most acute structural abnormalities, a number of subacute spinal pathologies may be much more difficult to detect. Spinal cord infarction can be challenging to confirm in the acute phase and repeated spinal imaging is often performed to ascertain the diagnosis. Subtle changes following whiplash injury are not always readily visualized despite clinical evidence of myelopathy [8].

In sharp contrast with the above acute conditions, spinal imaging has relatively limited value in confirming progressive neurodegenerative cord pathologies, such as spinal muscular atrophy, primary lateral sclerosis (PLS), hereditary spastic paraplegia, dorsal root ganglionopathy, HTLV-1 associated myelopathy, amyotrophic lateral sclerosis (ALS), spinocerebellar ataxia or HIV vacuolar myelopathy.

The meaningful interpretation of cord atrophy is notoriously difficult and remains largely subjective in the absence of quantitative metrics. Qualitative cues may be nonspecific and require careful appraisal. The specificity and sensitivity of 'owl's eyes' or 'snake's eye' signs on axial imaging is relatively poor but may indicate cord infarction, PLS or ALS in the appropriate clinical context [9]. Similarly, the 'inverted V sign' or hyperintensities of the dorsal columns is nonspecific and may be observed in subacute combined degeneration of the cord, copper deficiency myeloneuropathy and tabes dorsalis. Inferior cervical cord atrophy, anterior shifting of the posterior wall of the dural canal and enlarged posterior epidural compartment observed in flexion MR studies may indicate Hirayama disease, but similar findings may also be observed following trauma $[10,11]$.

\section{Future directions and novel imaging methods}

Patients presenting with slowly progressive spastic paraparesis or severe sensory ataxias often have limited findings on routine spinal imaging despite clinical evidence of a progressive myelopathy. Patients presenting with HIV myelopathies often present with significant clinical disability despite fairly nonspecific MRI findings. Patients with hereditary spastic paraparesis, HTLV infection, West Nile poliomyelitis all suffer from considerable myelopathies, yet may only exhibit nonspecific MR alterations on standard imaging [12]. ALS patients with tragically progressive spinal pathology only show subtle cord atrophy on routine spinal imaging $[13,14]$. PLS patients with considerable gait impairment may exhibit hyperintensities in the lateral corticospinal tracts but 
their gait impairment is often out of proportion to their imaging findings. Similarly, the diagnosis of dorsal root ganglionopathy may remain elusive despite progressive and selective cord atrophy [15]. In summary, a relatively large group of sinister and progressive spinal conditions exist where standard spinal MRI has remarkably little to offer. This is in striking contrast with the detection rates of advanced quantitative techniques used in a research setting.

In ALS, spinal MRS detects presymptomatic metabolic changes in mutation carriers [16], measures of cord atrophy correlate with clinical disability [17], diffusion tensor imaging (DTI) captures longitudinal changes [18], MRS correlates with clinical disability [19], and combined diffusion tensor and cross-sectional area measures have been repeatedly proposed as sensitive biomarkers of disease progression [20]. In traumatic neck injuries, DTI, high angular resolution diffusion-weighted imaging and magnetization transfer imaging show promise in capturing pathology [21]. While DTI primarily evaluates the longitudinal fibers of the cord, q-ball imaging promises to characterize the commissural and dorso-ventral fibers in the spinal cord [22]. In multiple sclerosis, magnetization transfer imaging [23], DTI fiber tractography [24], myelin water fraction estimation [25] and DTI metrics [26] have all been successfully correlated with clinical metrics. Spinal functional MRI has been applied both to multiple sclerosis [27] and chronic incomplete spinal cord injury [28]. The majority of quantitative spinal cord MRI studies are undertaken in ALS, MS and spinal cord injuries and very few dedicated imaging studies can be identified in hereditary spastic paraparesis and spinocerebellar ataxias [29].

\section{Conclusions}

The relentless methodological advances from pneumomyelography to cord tractography indicate that we are likely to witness continued progress in the field. Despite recent developments, an urgent and unmet need persists for sensitive spinal measures. These markers are indispensable for accurate clinical monitoring, pharmaceutical trial end points, and for the development of viable diagnostic and prognostic indicators.

\section{Financial \& competing interests disclosure}

$P$ Bede is supported by the Health Research Board (HRB - Ireland), the Irish Institute of Clinical Neuroscience (IICN) - Novartis Ireland Research Grant, The Iris O'Brien Foundation, The Perrigo Clinician-Scientist Research Fellowship, and the Research Motor Neuron (RMN-Ireland) foundation. The authors have no other relevant affiliations or financial involvement with any organization or entity with a financial interest in or financial conflict with the subject matter or materials discussed in the manuscript apart from those disclosed.

No writing assistance was utilized in the production of this manuscript.

\section{Role of the funding source}

The sponsors of the study had no role in study design, data analysis, data interpretation or writing of the report.

\section{Open access}

This article is distributed under the terms of the Creative Commons Attribution License 4.0 which permits any use, distribution, and reproduction in any medium, provided the original author(s) and the source are credited. To view a copy of the license, visit http://creativecommons.org/ licenses/by/4.0/

\section{References}

1 Dandy WE. Röntgenography of brain after the injection of air into the spinal canal. Ann. Surg. 70, 397-403 (1919).

2 Jacobaeus HC. On insufflation of air into the spinal canal for diagnostic purposes in cases of tumors of the spinal canal. Acta Med. Scand. 55, 555-564 (1921).

3 Sicard JA, Forestier JE. Methode generale d'exploration radiologique par l'huile iodée (Lipiodol ${ }^{\circledR}$ ). Bull. Soc. Med. Hop. Paris 46, 463-468 (1922).

4 Hoeffner EG, Mukherji SK, Srinivasan A, Quint DJ. Neuroradiology back to the future: spine imaging. Am. J. Neuroradiol. 33(6),
999-1006 (2012).

5 Heinz ER. Development of the C1-C2 puncture in neuroradiology: a historical note. Am. J. Neuroradiol. 26(1), 5-6 (2005).

6

Moniz E. L'encephalographie arterielle, son importance dans la localization des tumeurs cerebrales. Rev. Neurol. (Paris). 2, 72-90 (1927).

7 Walker J 3rd, El Abd O, Isaac Z, Muzin S. Discography in practice: a clinical and historical review. Curr. Rev. Musculoskelet. Med. 1(2), 69-83 (2008).

8 Anderson SE, Boesch C, Zimmermann $\mathrm{H}$ et al. Are there cervical spine findings at MR imaging that are specific to acute symptomatic whiplash injury? A prospective controlled study with four experienced blinded readers. Radiology 262(2), 567-575 (2012).

9 Lebouteux MV, Franques J, Guillevin R et al. Revisiting the spectrum of lower motor neuron diseases with snake eyes appearance on magnetic resonance imaging. Eur. J. Neurol. 21(9), 1233-1241 (2014).

10 Bede P, Walsh R, Fagan AJ, Hardiman O. 'Sand-watch' spinal cord: a case of inferior cervical spinal cord atrophy. J. Neurol. 261(1), 235-237 (2013).

11 Bede P, Bokde AL, Byrne SC, Elamin M, Walsh RJ, Hardiman O. Waterskier's Hirayama syndrome. J. Neurol. 258(11), 2078-2079 (2011). 
12 Ali M, Safriel Y, Sohi J, Llave A, Weathers $S$. West Nile virus infection: MR imaging findings in the nervous system. Am. J. Neuroradiol. 26(2), 289-297 (2005).

13 Bede P, Bokde AL, Byrne S, Elamin M, Fagan AJ, Hardiman O. Spinal cord markers in ALS: diagnostic and biomarker considerations. Amyotroph. Lateral. Scler. 13(5), 407-415 (2012).

14 Bede P, Hardiman O. Lessons of ALS imaging: Pitfalls and future directions - a critical review. NeuroImage. Clinical 4 , 436-443 (2014).

15 Abkur TM, Bede P. Clinical reasoning: reversible gait ataxia: from wheelchair to independent mobility. Neurology 88(15), e145-e149 (2017).

16 Carew JD, Nair G, Andersen PM et al. Presymptomatic spinal cord neurometabolic findings in SOD1-positive people at risk for familial ALS. Neurology 77(14), 1370-1375 (2011).

17 Cohen-Adad J, El Mendili MM, MorizotKoutlidis $\mathrm{R}$ et al. Involvement of spinal sensory pathway in ALS and specificity of cord atrophy to lower motor neuron degeneration. Amyotroph Lateral Scler Frontotemporal Degener. 14(1), 30-38 (2013).
18 Agosta F, Rocca MA, Valsasina P et al. A longitudinal diffusion tensor MRI study of the cervical cord and brain in amyotrophic lateral sclerosis patients. J. Neurol. Neurosurg. Psychiatry 80(1), 53-55 (2009).

19 Ikeda K, Murata K, Kawase Y et al. Relationship between cervical cord $1 \mathrm{H}$-magnetic resonance spectroscopy and clinoco-electromyographic profile in amyotrophic lateral sclerosis. Muscle Nerve 47(1), 61-67 (2013).

20 El Mendili MM, Cohen-Adad J, PelegriniIssac $\mathrm{M}$ et al. Multi-parametric spinal cord MRI as potential progression marker in amyotrophic lateral sclerosis. PLoS One 9(4), e95516 (2014).

21 Cohen-Adad J, El Mendili MM, Lehericy S et al. Demyelination and degeneration in the injured human spinal cord detected with diffusion and magnetization transfer MRI. Neuroimage 55(3), 1024-1033 (2011).

22 Cohen-Adad J, Descoteaux M, Rossignol S, Hoge RD, Deriche R, Benali H. Detection of multiple pathways in the spinal cord using q-ball imaging. Neuroimage 42(2), 739-749 (2008).

23 Kearney H, Altmann DR, Samson RS et al. Cervical cord lesion load is associated with disability independently from atrophy in MS.
Neurology 84(4), 367-373 (2015).

24 Hodel J, Besson P, Outteryck O et al. Pulse-triggered DTI sequence with reduced FOV and coronal acquisition at $3 \mathrm{~T}$ for the assessment of the cervical spinal cord in patients with myelitis. Am. J. Neuroradiol. 34(3), 676-682 (2013).

25 Laule C, Vavasour IM, Zhao Y et al. Two-year study of cervical cord volume and myelin water in primary progressive multiple sclerosis. Mult. Scler. 16(6), 670-677 (2010).

26 Oh J, Sotirchos ES, Saidha $\mathrm{S}$ et al. Relationships between quantitative spinal cord MRI and retinal layers in multiple sclerosis. Neurology 84(7), 720-728 (2015).

27 Valsasina P, Rocca MA, Absinta M et al. Cervical cord FMRI abnormalities differ between the progressive forms of multiple sclerosis. Hum. Brain Mapp. 33(9), 2072-2080 (2012).

28 Cadotte DW, Bosma R, Mikulis D et al. Plasticity of the injured human spinal cord: insights revealed by spinal cord functional MRI. PLoS One 7(9), e45560 (2012).

29 Martins CR Jr, Martinez AR, De Rezende TJ et al. Spinal cord damage in spinocerebellar ataxia type 1. Cerebellum doi:10.1007/ s12311-017-0854-9 (2017) (Epub ahead of print). 